Eduardo Dei Cas

\section{Benoît Soulez}

François Palluault

Pierre Charet

Daniel Camus

\section{ADRESSE}

E. Dei Cas : maître de conférences-praticien hospitalict au service de parasitologie-mycologie du centre hospitalier universitaire de Lille. B. Soulez: interne de spécialité au centre haspitalier Universitaire de Lille. F. Palluault : chargé de cours à l'université de Lille I. P. Charet : professeur de biochimie à l'université de Lille I. D. Camus : professeur de parasitologie à l'université de Lille II, directeur de l'unité 42 de l'Inserm. Inserm U. 42, 369, rue Jules-Guesde, 59650 Villeneuve-d'Ascq, France.

$\mathrm{m} / \mathrm{s} n^{\circ} 6$ vol. 6 , juin 90

\title{
Pneumocystis carinii, un défi pour le biologiste
}

Pneumocystis carinii est un parasite de taxonomie incertaine, possédant certaines caractéristiques des protozoaires et d'autres des levures ou autres champignons unicellulaires. Il vit normalement comme un saprophyte des alvéoles pulmonaires de plusieurs espèces de mammifères, dont l'homme. En cas de baisse des défenses immunologiques de l'organisme, $P$. carinii est responsable d'une affection opportuniste sévère, la pneumocystose, dont la symptomatologie est celle d'une pneumonie interstitielle. Cette maladie est la cause du décès de $25 \%$ des malades atteints de SIDA. Différents modèles animaux, ainsi que le développement encore débutant de méthodes de culture, ont permis de mieux connaître certains aspects de la commutation saprophyte/pathogène du parasite. Beaucoup reste cependant encore à faire dans le domaine de la biologie moléculaire et cellulaire de cet agent qui est devenu, depuis le début des années 80 , un problème essentiel de santé publique.

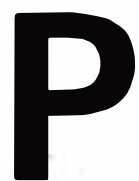

neumocystis carinii est un important agent de pneumopathie grave chez le patient immunodéprimé. C'est une épidémie de pneumiocystose aux États-Unis, en 1981, qui a révélé le syndrome d'immunodéficience acquise (SIDA). Ce parasite est retrouvé fréquemment à l'état saprophyte dans le poumon de l'homme et d'autres mammifères sains [1] : La connaissance des facteurs impliqués dans la transition saprophyte-pathogène de $P$. carinii est essentielle pour comprendre le développement et la physiopathologie de la pneumocystose et éventuellement pour la prévenir.

Les animaux de laboratoire étant souvent naturellement parasités par
$P$. carinii, l'induction d'un état d'immunodépression révèle l'infection latente. Différentes techniques de culture in vitro ont été proposées [2-4], mais les résultats sont modestes et le cycle complet ne semble pas avoir lieu dans ces systèmes. Le développement de techniques d'extraction de parasites vivants à partir du poumon de l'hôte, l'obtention des différents stades parasitaires in vitro et la connaissance des besoins métaboliques de $P$. carinii demeurent des objectifs à atteindre.

$P$. carinii a été décrit en 1909 par Carlos Chagas [5, vol 1, p. 1-7] dans des poumons de cobaye et peut-être d'humains infestés par des trypanosomes. Antonio Carini, en 1910, le retrouve chez des rats infestés par 


\section{Reconstruction 3-D d'un trophozoïte de pneumocystis carinii}

L'étude en microscopie électronique à transmission de coupes sériées permet de mieux comprendre la cytologie de Pneumocystis carinii. La superposition des plans de coupe successifs nous a permis d'entreprendre la reconstruction tridimensionnelle des différents stades parasitaires a l'aide de l'outil informatique. Cette démarche (en cours) a permis notamment de préciser la structure de l'appareil de Golgi, de la mitochondrie el surtout du réticulum endoplasmique du trophozoïle. La figure A montre une image ultrastructurale de ce stade parasitaire. Le noyau, bien visible, présente un nucléole excentré. A partir de la membrane externe de l'enveloppe nucléaire se développe le réticulum endoplasmique. La mitochondrie, à crêtes lamellaires, montre une expansion (pointe de flèche) et occupe un volume important dans le cytoplasme. Deux petites vésicules golgiennes sont visibles (fleches). La membrane plasmique s'évagine en formant des filopodes ou prolongements tubulaires au nombre de trois sur ce plan. Une couche externe dense épouse le contour de celte membrane.

Dans la figure B une reconstruction 3-D partielle à partir de coupes sériées $d u$ mème trophozoïle est présentée. Sur celle image "en miroir " de la figure A, une hémi-surface parasitaire (en vert) a été enlevée pour montrer le noyeau (en rouge), le réticulum endoplasmique (dans la même couleur) et la mitochondrie (en bleu).

Abréviations : $\mathrm{Mi}=$ mitochondrie; $\mathbf{N}=$ noyau ; $\mathbf{N u}=$ nucléole; $\mathbf{R E G}=$ réticulum endoplasmique granulaire; REL $=$ réticulum endoplasmique lisse.
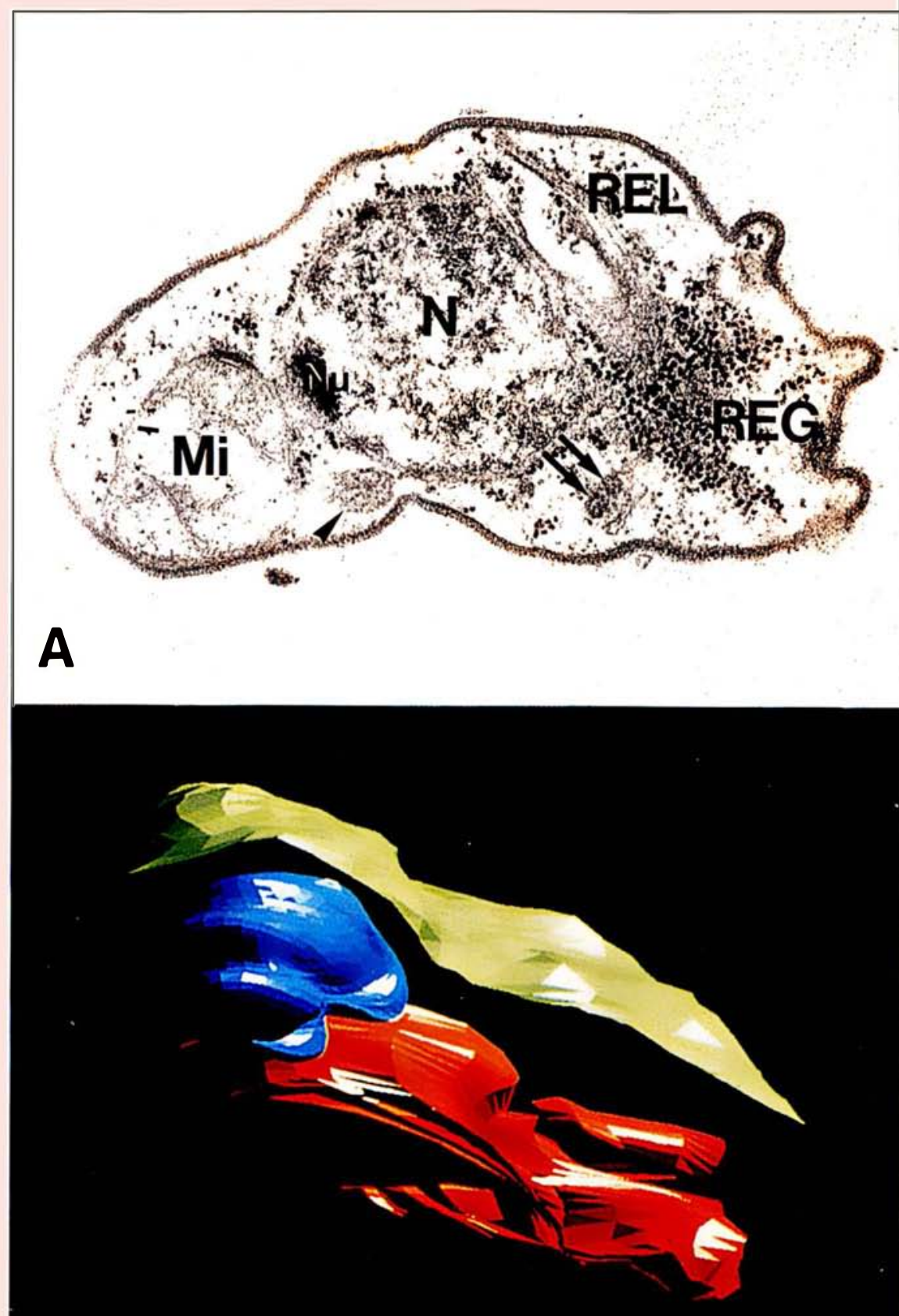

B 
Trypanosoma lewisi. Les deux auteurs ont cru que les formes kystiques qu'ils avaient découvertes correspondaient à un nouveau stade dans le cycle biologique des trypanosomes. Deux années plus tard, P. Delanoë et M. Delanoë [6], dans un remarquable article de trois pages, rapportent la présence du parasite chez le rat non infesté par Trypanosoma spp, décrivent l'organisme comme une nouvelle entité, affirment son tropisme pulmonaire et le nomment Pneumocystis carinii, en suggérant l'existence d'affinités avec les coccidies.

En 1942, ce parasite a été retrouvé chez l'homme en Hollande par Van der Meer et Brug; mais il fut établi, en 1952 seulement, par Vanek et Jirovec, que $P$. carinii était l'agent d'une pneumonie interstitielle à plasmocytes décrite chez des enfants prématurés et malnutris en Europe dès les années 30 [7]. Plus tard, P. carinii a été reconnu comme un important agent de pneumonie grave, mortelle en absence de traitement, chez les patients naturellement ou iatrogéniquement immunodéprimés.

A partir de 1981, la pneumopathie à $P$. carinii (PPC) devient la parasitose la plus importante au cours du syndrome d'immunodéficience acquise (SIDA). Soixante à $80 \%$ des patients font une PPC au cours de l'évolution de leur infection: cette pneumonie est la cause principale du décès chez $25 \%$ de ces patients $[5$, vol. II, p. 30 ; 8]. De plus, cette parasitose a une importance considérable en dehors du contexte du SIDA. Durant la dernière année nous avons enregistré, au centre hospitalier de Lille, une augmentation très importante du nombre de patients développant une PPC en dehors de toute infection par le virus de l'immunodéficience humaine (HIV). Avant 1979, la plupart des cas de PPC aux États-Unis étaient observés chez des patients cancéreux ou receveurs de greffes d'organe [9]. Enfin il faut ajouter que la PPC est occasionnellement rapportée chez l'hôte immunocompétent [10].

\section{Biologie, cycle vital et aspects taxonomiques}

Pneumocystis carinii est un microorganisme eucaryote. Tous les stades $\mathrm{m} / \mathrm{s} n^{\circ} 6$ vol. 6 , juin 90

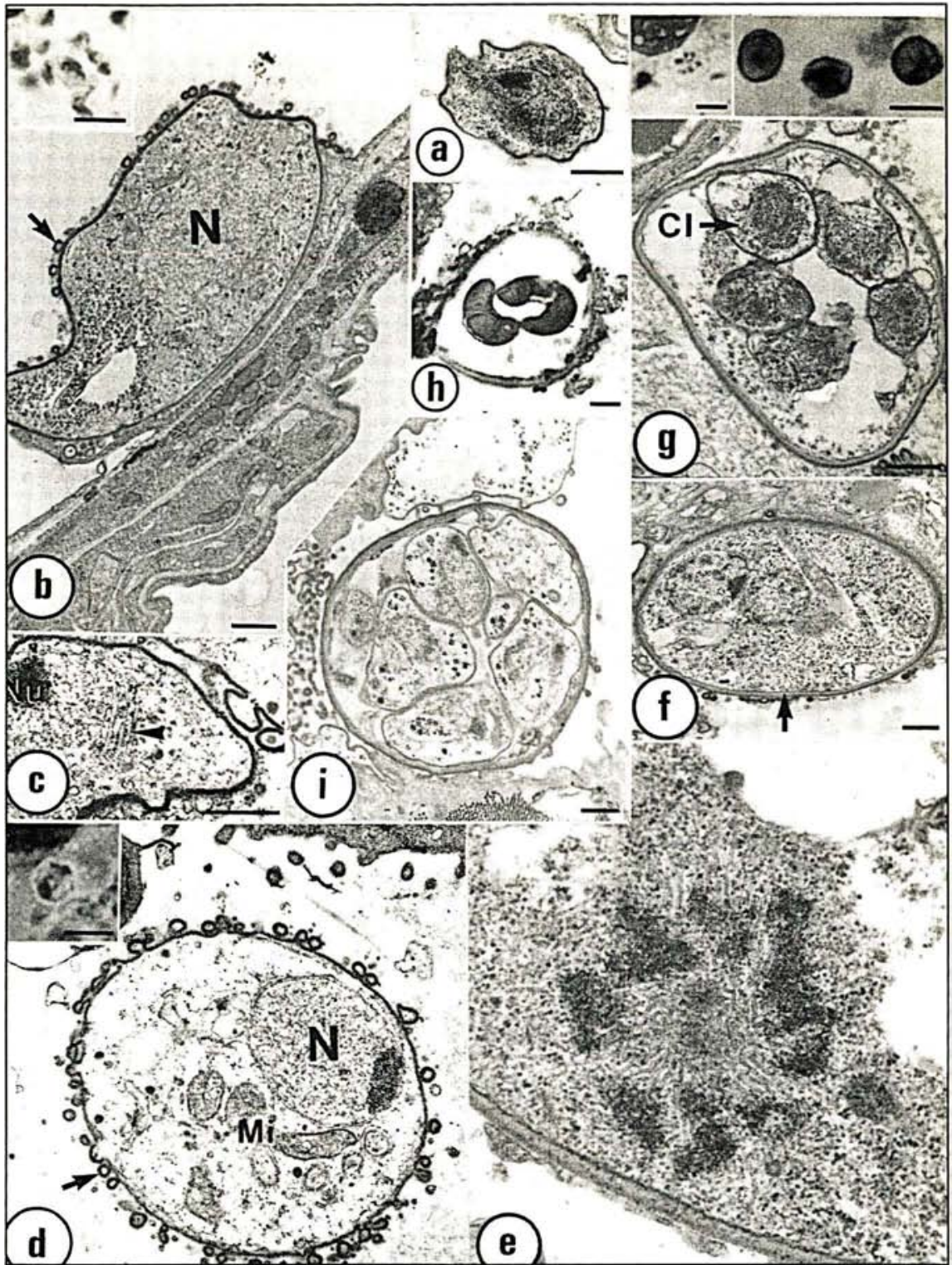

Figure 1. Morphologie et cycle biologique de Pneumocystis carinii. a) Tropozoïte améboïde de petite taille. La membrane plasmique est surmontée d'une couche dense aux électrons qui accentue le contour du parasite; b) trophozoïte attaché à une cellule alvéolaire. La zone d'attachement est bien visible ainsi que les filopodia en section transversale (flèche). Le contour apparaît foncé grâce à l'utilisation du rouge de ruthénium, un colorant des groupements anioniques de glucanes. Encart : trophozoïtes colorés au RAL-555, coloration panoptique; c) un autre trophozoïte avec un réticulum endoplasmique granulaire bien visible (pointe de flèche); d) prékyste précoce, uninucléé, résultant de l'évolution des trophozoïtes. Les filopodia sont encore nombreux (flèche) (cultures sur explant de poumon). Encart : prékyste intermédiaire coloré au RAL-555; e) prékyste intermédiaire : division nucléaire bien visible ; chromosomes (zones denses) et microtubules du fuseau au centre. La paroi montre une couche moyenne peu dense aux électrons; f) prékyste intermédiaire binucléé ; paroi caractéristique (flèche); g), h) et i) trois aspects de kystes matures contenant des corps intrakystiques bien individualisés. Encart sur la figure $g$ : kyste mature coloré au RAL-555 (gauche); formes kystiques colorées au bleu de toluidine $O$ (droite). Hôte expérimental : lapin. Sur les images d'ultrastructure, barre $=0,5 \mu \mathrm{m}$; sur les images de microscopie photonique, barre $=10 \mu \mathrm{m}$. Abréviations : $\mathrm{Cl}$ : corps intrakystique; $\mathrm{Mi}$ : mitochondrie ; $\mathrm{N}$ : noyau; $\mathrm{Nu}$ : nucléole. 


\section{RÉFÉRENCES}

1. Walzer PD. Experimental models of Preumocystis carinii infections. In : Young LS. Pneumocystis Carinii Pneumonia. New York: Marcel Dekker, 1984: 7-76.

2. Cushion MT. In vitro studies of Pneumocystis carinii. J Protozool $1989 ; 36: 45-52$

3 . Tegoshi T. New system of in vitro cultivation of Pneumocystis carinii without feeder cells. J Kyoto Pref Univ Med 1988; 97 1473-82

4. Dei-Cas E, Soulez B, Camus D. Ultrastructural study of Pneumocystis carinii in explant cultures of rabbit lung and in cultures with and without feeder cells. $J$ Protozool 1989 ; 36 : 55-7.

5. Hughes WT. Pneumocystis Carinii Pneumonitis. Boca Raton: CRC Press, 1987.

6 . Delanoë $P$, Delanoë M. Sur les rapports des kystes de carinii des poumons de rats avec le Trypanosoma lewisi. CR Acad Sci (Paris) 1912 ; $155:$ 658-60.

7. Walzer PD. Historical perspectives on Pneumocystis carinii. J Protozool $1989 ; 36$ : 39-41.

8. Kovacs JA, Masur H. Pneumocystis carinii pneumonia : therapy and prophylaxis. $J$ Infect Dis 1988 ; 158 : 254-9.

9. Masur H. Clinical studies of Pneumocystis carinii and relationships to AIDS. $J$ Protozool 1989 ; $36: 70-4$.

10. Young LS. Clinical aspects of pneumocystosis in man : epidemiology, clinical manifestations, diagnostic approaches, and sequelae. In : Young LS. Pneumocystis Cari nii Pneumonia. New York: Marcel Dekker, 1984: 139-74.

11. Matsumoto Y, Yoshida Y. Sporogony in Pneumocystis carinii : synaptonemal complexes and meiotic nuclear division observed in precysts. J Protozool 1984; 31 : 420-8.

12. Kovacs JA, Allegra CJ, Beaver J, et al. Characterization of de novo Folate synthesis in Pneumocystis carinii and Toxoplasma gondii : potential for screening therapeutic agents. Infect Dis $1989 ; 160$ : 312-20.

13. Matsumoto Y, Matsuda S, Tegoshi T. Yeast glucan in the cyst wall of Pneumocystis carinii. J Protozool 1989 ; 36 : 21-2.

14. Ruffolo JJ, Cushion MT, Walzer PD. Ultrastructural observations on life cycle stages of Pneumocystis carinii. J Protozool 1989 ; $36: 53-4$.

15. Edman JC, Kovacs JA, Masur H, Santi DV, Elwood HJ, Sogin M. Ribosomal RNA sequence shows Pneumocystis carinii to be a member of the fungi. Nature $1988 ; 334: 519-22$.

16. Stringer SL, Stringer JR, Blase MA, Walzer PD, Cushion MT. Pneumocystis carinii : sequence from ribosomal RNÁ implies a close relationship with fungi. Exp Parasi- connus du parasite ont été observés dans les alvéoles pulmonaires de l'homme et des animaux. On distingue clairement (figure 1, page précédente et Tableau I) :

a) les formes végétatives ou trophozoïtes, uninucléées, améboïdes, mesurant 2 à $8 \mu \mathrm{m}$ de diamètre, qui présentent une pellicule formée par la membrane cellulaire et une couche externe dense aux électrons avec des sites polysaccharidiques qui réagissent avec la concanavaline A. L'encart de la page 518 montre en détail l'ultrastructure de ce stade parasitaire et sa reconstruction tridimensionnelle ;

b) les prékystes, ovoïdes, mesurant 3 à $6 \mu \mathrm{m}$ de diamètre ; d'abord uninucléés, avec une pellicule semblable à celle du trophozoïte (prékyste précoce). Plus tard ces formes deviennent multinucléées (jusqu'à 8 noyaux) et développent une pellicule ou paroi cellulaire " à trois couches ": la membrane cellulaire, une couche intermédiaire peu dense aux électrons et une couche externe dense (prékyste intermédiaire et tardif). Dans ces deux dernières couches, chez les prékystes provenant de poumons de lapin, il existe des sites polysaccharidiques qui réagissent aussi avec la concanavaline A ;

c) les kystes, mesurant 4 à $6 \mu \mathrm{m}$ de diamètre, avec une paroi similaire à celle des prékystes intermédiaires et tardifs et contenant huit corps intrakystiques bien individualisés, uninucléés qui deviendront des trophozoïtes.

La forme infectante demeure inconnue. Probablement s'agit-il des corps intrakystiques, appelés parfois "sporozoïtes".

Matsumoto et Yoshida [11], à partir de l'observation de complexes synaptonémaux* dans le prékyste précoce, ont proposé un cycle hypothétique au cours duquel ce stade subit une division méiotique conduisant à la formation des 8 corps intrakystiques haploïdes. Une fusion

\footnotetext{
* Complexe synaptoménal: structure des régions d'appariement des chromosomes au cours de la méiose, visible en microcopie électronique aux stades zygotène et pachytène.
}

nucléaire des trophozoïtes haploïdes restituerait l'état diploïde.

Sur le plan taxonomique, $P$. carinii demeure une énigme. L'aspect améboïde du trophozoïte et la présence de prolongements cytoplasmiques ou filopodia (figure 1) permettent de le rapprocher des protozoaires. Par ailleurs, $P$. carinii est sensible à des médicaments efficaces contre des protozoaires parasites comme la pentamidine et l'association triméthoprimesulfaméthoxazole (TMP-SMZ). Comme Toxoplasma gondii, il est capable de synthétiser de novo des folates à partir de l'acide paraaminobenzoïque (PABA) [12]. En revanche, les affinités tinctoriales et la structure de la paroi des formes kystiques évoquent celles de la paroi fongique [13]. La présence de crêtes mitochondriales lamellaires permettrait de le rapprocher des fungi [14]. Par son ARN ribosomial, P. carinii serait très proche de Saccharomyces cerevisiae $[15,16]$. De plus, tandis que chez les protozoaires étudiés jusqu'à maintenant les enzymes thymidylate synthétase (TS) et dihydrofolate réductase (DHFR) existent comme une même protéine bifonctionnelle, chez $P$. carinii ces enzymes sont deux chaînes polypeptidiques séparées et monofonctionnelles dont la synthèse est codée par des gènes se trouvant sur des chromosomes différents [17]. Des anticorps monoclonaux antimannanes de $S$. cerevisiae réagissent contre $P$. carinii (résultats non publiés). Il existe des affinités immunologiques entre $P$. carinii, $S$. cerevisiae et d'autres espèces fongiques.

Cependant, de nombreux protozoaires (cryptomonadines, eugléniens, zooflagellés) ont aussi des mitochondries à crêtes lamellaires $[18, p .63]$ et bien que chez les champignons pathogènes les crêtes mitochondriales soient souvent lamellaires, dans d'autres groupes fongiques elles sont tubulaires [19]. L'absence apparente d'ergostérol dans la membrane de $P$. carinii [20] pourrait expliquer sa résistance à l'amphotéricine $\mathrm{B}$, un antifongique majeur, et constitue un argument contre sa nature fongique. De plus, $P$. carinii ne se développe pas in vitro dans des milieux de culture pour champignons. Enfin, par la structure de son ARNr 5S, P. carinii serait très proche du groupe Rhizo- 
Tableau I

STADES PARASITAIRES DE PNEUMOCYSTIS CARINII

EN MICROSCOPIE PHOTONIQUE ET ÉLECTRONIQUE

\begin{tabular}{|c|c|c|c|c|c|}
\hline & Trophozoïte & Précoce & $\begin{array}{c}\text { Prékyste } \\
\text { Intermédiaire }\end{array}$ & Tardif & Kyste \\
\hline $\begin{array}{l}\text { MP(1) } \\
\text { BTO* } \\
\text { RAL555** }\end{array}$ & $\begin{array}{l}- \\
+\end{array}$ & $\overline{+}$ & $\begin{array}{l}+ \\
+\end{array}$ & $\begin{array}{l}+ \\
+\end{array}$ & $\begin{array}{l}+ \\
+\end{array}$ \\
\hline $\begin{array}{l}\text { ME(2) [11] } \\
\text { Forme } \\
\text { Noyaux } \\
\text { Complexe } \\
\text { synaptonémal } \\
\text { OAN**** } \\
\text { Fuseaux de division } \\
\text { Mitochondries } \\
\text { Paroi } \\
\text { Filopodia }\end{array}$ & $\begin{array}{c}\text { irrégulière } \\
1 \\
\text { Non } \\
\text { Non } \\
\text { Non } \\
\text { allongées } \\
2 \text { couches } \\
20-30 \mathrm{~nm} \\
\text { nombreux }\end{array}$ & $\begin{array}{c}\text { ovoïde } \\
1 \quad(2 \mu \mathrm{m}) \\
\text { Oui } \\
\text { Non } \\
\text { Non } \\
\text { groupées } \\
2 \text { couches } \\
40-50 \mathrm{~nm} \\
\text { quelques }\end{array}$ & $\begin{array}{c}\text { ovoïde } \\
2-4 \\
\\
\text { Non } \\
\text { Oui } \\
\text { Oui } \\
\text { groupées } \\
3 \text { couches } \\
100 \mathrm{~nm} \\
\text { quelques }\end{array}$ & 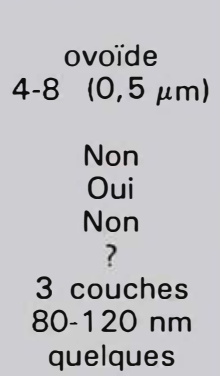 & $\begin{array}{c}\text { ovoïde } \\
1^{* * *} \\
\text { Non } \\
\text { Non } \\
\text { Non } \\
1^{* * *} \\
3 \text { couches } \\
\text { épaisses } \\
\text { quelques }\end{array}$ \\
\hline
\end{tabular}

* Bleu de toluidine $O$ : coloration métachromatique des formes à paroi à trois couches. ** Coloration panoptique : paroi à 3 couches non colorée (halo clair). *** Dans chaque corps intrakystique. *** Organite associé au noyau. (1) MP: microscopie photonique ; (2) ME : microscopie électronique.

poda/Myxomycota/Zygomycota, appelé provisoirement "Protista fungi" par Watanabe et al. [21]. Pour ces auteurs $P$. carinii n'est pas phylogénétiquement en relation avec des ascomycètes ou basidiomycètes "communs" ni avec des protozoaires.

En définitive, la position taxonomique de $P$. carinii n'est pas encore établie, d'une part parce que l'avis des différents auteurs dépend de la valeur taxonomique qu'ils attribuent aux divers caractères et d'autre part parce que les critères d'inclusion d'un organisme parmi les champignons ou parmi les protozoaires n'ont pas été précisément définis [22]. Toutefois, la position taxonomique d'un organisme n'est généralement pas établie sur un seul critère mais résulte de l'association de caractères morphologiques, biochimiques, physiologiques, immunologiques, génétiques et écologiques. Ainsi, il est certain qu'on ne peut pas tenir compte uniquement des homologies de séquence de l'ARNr pour classer Pneumocystis [22].

La question dépasse le domaine taxonomique et à des incidences pratiques évidentes car les protozooses et les mycoses humaines sont remarqua$\mathrm{m} / \mathrm{s} n^{\circ} 6$ vol. 6 , juin 90 blement différentes sur les plans clinique, thérapeutique et épidémiologique.

\section{Modèles expérimentaux}

Pneumocystis carinii a été retrouvé dans le poumon de nombreux mammifères (marsupiaux, rongeurs, édentés, lagomorphes, carnivores, ongulés, primates, etc. [5, vol. I, p. 72-3]). Cependant, seuls des rats, des souris $[5$, vol. $I$, p. 71$]$, des lapins [23, 24] et des furets [25] ont été utilisés comme hôtes expérimentaux. Le rat et la souris, utilisés le plus souvent, soumis à une corticothérapie prolongée ou athymiques, développent une importante pneumopathie à $P$. carini en deux à trois mois. Étant naturellement infectés par $P$. carinii l'inoculation du parasite n'est pas nécessaire. Ces modèles animaux ont permis la mise au point des protocoles thérapeutiques actuels, le développement de recherches sur la biologie de $P$. carinii et des études physiopathologiques.

Récemment nous avons décrit chez le lapin une pneumocystose spontanée au moment du sevrage qui se développe en absence d'immunodépres- sion induite [24]. Pratiquement tous les lapereaux sevrés, provenant d'élevages de plusieurs régions, sont intensément parasités par $P$. carinii et développent une pneumonie d'importance variable (figure 2, p. 522) associée à des perturbations des lipides sériques, de l'activité lacticodéshydrogénase (LDH) et sans réponse humorale détectable. Dans les semaines suivant le sevrage les animaux guérissent spontanément et la réduction progressive du taux de parasitisme (figure 3, p. 523) est corrélée à l'apparition d'anticorps spécifiques (figure 4, p. 524).

Les premières tentatives de culture in vitro datent de 1977 [2]. La plupart des auteurs rapportent le développement du parasite en co-culture avec des lignées primaires ou continues de cellules nourricières. La culture axénique a aussi été rapportée [3]. Dans ces systèmes, l'inoculum est constitué par des parasites séparés du tissu pulmonaire. Or, les techniques de séparation peuvent entraîner des altérations de $P$. carinii, ce qui constitue un obstacle important aux études in vitro [4]. Avec ou sans cellules nourricières, la culture est en général maintenue moins de 8 jours. Le parasite 


\section{RÉFÉRENCES}

17. Edman U, Edman JC, Lundgren B, Santi DV. I solation and expression of the Pneumocystis carinii thymidylate synthase gene. Proc Natl Acad Sci 1989 ; 86 : 6503-7. 18. De Puytorac P, Grain J, Mignot J-P. Précis de protistologie. Paris : éd Boubée, 1987. 19. Garrison RG. Ultrastructural gytology of pathogenic fungi. In : Howard $\mathrm{DH}$, ed. Fungi Pathogenic for Humans and Animals. New York : Marcel Dekker, Inc, 1983 : 229-321. 20. Yoshikawa H, Morioka H, Yoshida Y. Freeze fracture localization of filipinsterol complexes in plasma- and cytomembranes of Pneumocystis carinii. J Protozool 1987 ; 34 : 131.

21. Watanabe J, Hori $H$, Tanabe $K$, Nakamura Y. Phylogenetic association of Pneumocystis carinii with the "Rhizopoda/Myxomycota/Zygomycota group " indicated by comparison of $5 \mathrm{~S}$ ribosomal RNA sequences. Mol Biol Parasitol 1989 ; 32: 163-8.

22. Hughes WT. Pneumocystis carinii : taxing taxonomy. Eur J Epidemiol 1989 ; 5 : 265-9. 23. Soulez B, Dei-Cas E, Camus D. Le lapin, hôte expérimental de Pneumocystis carinii. Ann Parasitol Hum Comp 1988; 63 : 5-15.

24. Soulez B, Dei-Cas E, Charet P, Mougeot $G$, Caillaux M, Camus D. The young rabbit : a nonimmunosuppressed model for Pneumocystis carinii pneumonia. J Infect Dis $1989 ; 160: 355-6$

25. Gigliotti F, Hughes WT. Passive immunoprophylaxis with specific monoclonal antibody confers partial protection against Pneumocystis carinii pneumonitis in, animal models. J Clin Invest $1988 ; 81$ 1666-8.

26. Sheehan PM, Stokes DC, Yeh YY, Hughes WT. Surfactant phospholipids and lavage phospholipase A2 in experimental Pneumocystis carinii pneumonia. Am Rev Respir Dis 1986 ; 134: 526-31.

27. Kernbaum S, Masliah J, Alcindor LG Bouton C, Christol D. Phospholipase activities of bronchoalveolar lavage fluid in rat Pneumocystis carinii pneumonia. $\mathrm{Br} J$ Exp Pathol 1983 ; 64 : 75-80.

28. Ernst P, Chen M-F, Wang N-S, Cosio M. Symbiosis of Pneumocystis carinii and cytomegalovirus in a case of fatal pneumonia. Can Med Assoc J 1983 ; 128 : 1089-92. 29. Charet P, Dei-Cas E, Soulez B, Camus D, Leibowitch J, Saimot AG. Bilan lipidique et TNF (Tumor necrosis factor) chez les sujets $\mathrm{VIH}^{+}$. Méd Mal Infect 1988; 18 : 772 .

30. Graves DC. Immunological studies of Pneumocystis carinii. J Protozool 1989; 36 : 60-9.

31. Blumenfeld W, Mandrell RE, Jarvis GA, Griffiss M. Localization of host immunoglobulin G to the surface of Pneumocystis carinii. Infect Immun 1990; 58 : 456-63. 32. Furuta TK, Ueda SK, Fujiwara K. Effect of T-cell transfer on Pneumocystis carini infection in nude mice. Jpn $J$ Exp Med $1984 ; 54: 57-64$

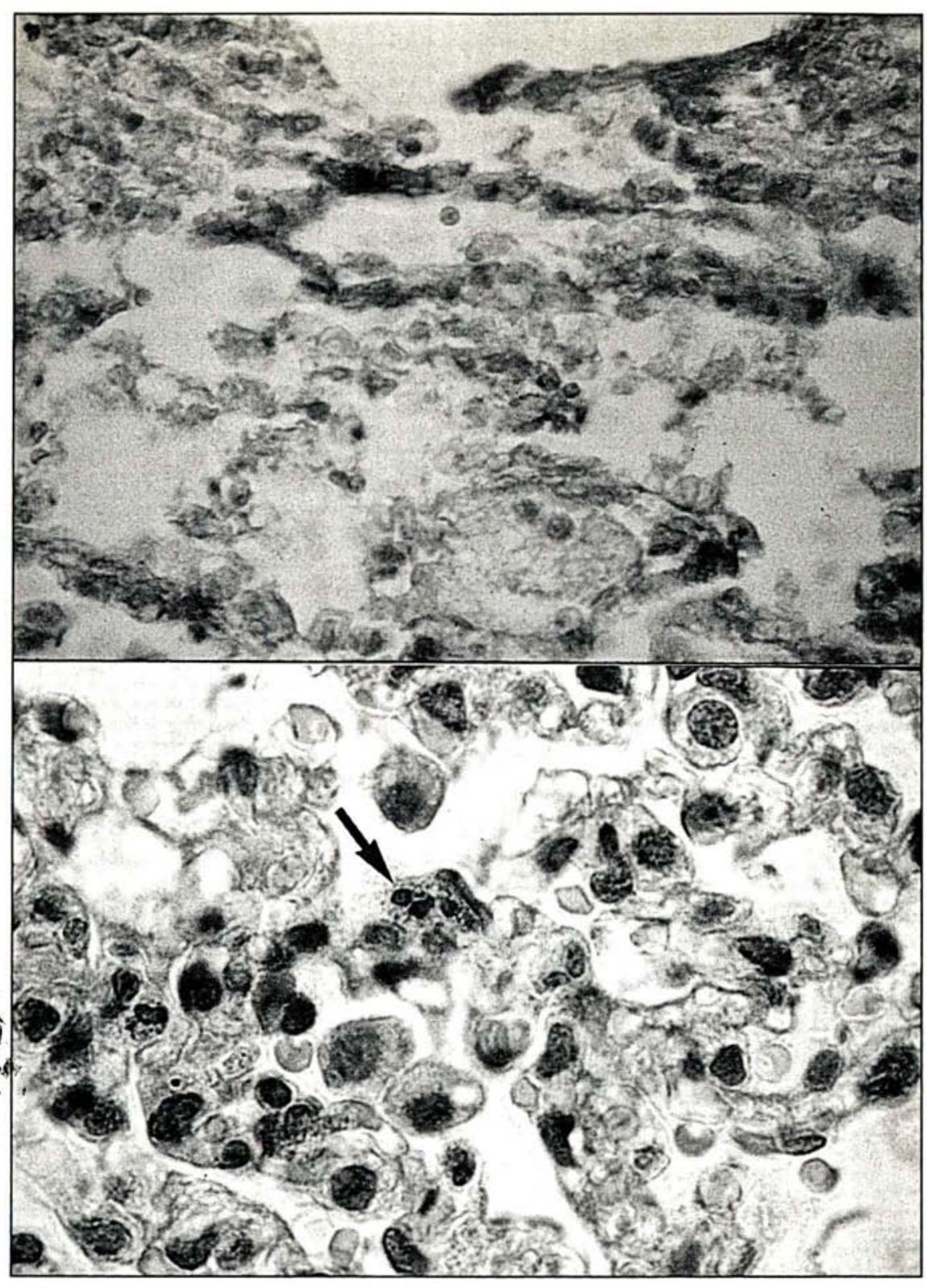

Figure 2. Histopathologie de la pneumonie à Pneumocystis cariniî. En haut : formes kystiques de $\mathrm{P}$. carinii colorées en violet par le bleu de toluidine $0, x 1000$. En bas: épaississement des cloisons alvéolaires, infiltrat macrophagique et éosinophilique (flèche). col. HES, x 3150. Hôte expérimental : lapin. La fibrose interstitielle rapportée chez le rat, surtout à l'arrêt des corticoides, et chez l'homme ne semble pas exister chez le lapin.

serait métaboliquement actif seulement pendant les premiers jours. il existe alors une augmentation du nombre de parasites due essentiellement à la multiplication des trophozoïtes. L'incorporation de PABA par $P$. carinii in vitro a aussi lieu seulement pendant les deux ou trois premiers jours de culture, en présence ou en absence de cellules nourricières [12]. La culture en continu n'est pas encore possible et les techniques pour suivre qualitativement et quantitativement l'évolution du parasite in vitro sont loin d'être parfaites.

Nous avons proposé une méthode de culture de $P$. carinii sur des explants pulmonaires [4]. Ce système s'avère 
intéressant. Nous avons suivi ces cultures à l'aide de la microscopie électronique à transmission (MET) et tous les stades parasitaires furent observés. Malgré les difficultés de la quantification, une augmentation de deux à dix du nombre total de parasites a été notée. Cette croissance, qui a lieu pendant les premiers jours, serait due à l'augmentation du nombre de trophozoïtes et de prékystes précoces (figure 1), mais aussi à la libération des corps intrakystiques contenus dans les kystes matures présents au départ dans l'explant. Le développement semble se bloquer au stade prékyste précoce.

La culture d'explants permet d'éviter les techniques de séparation de $P$. carinii des cellules de l'hôte, car on " ensemence " directement des fragments de poumon parasité. De plus, si le parasite se développe en général modérément, les explants constituent une source de $P$. carinii normal sur le plan ultrastructural, utilisable pendant plusieurs semaines [4]. Néanmoins, le parasite reste intimement attaché au tissu pulmonaire, ce qui rend difficile l'évaluation précise de la croissance et l'obtention de quantités importantes de parasites dépourvus de cellules de l'hôte, essentielle pour des études immunologiques, biochimiques et de biologie moléculaire.

\section{Rapports hôte-parasite}

$P$. carinii se trouve souvent à l'état saprophyte chez l'homme et chez d'autres mammifères. La prolifération parasitaire et la transformation saprophyte-pathogène de $P$. carinii exigent d'importantes perturbations des lymphocytes T [5, vol. I, p. 71]. Chez les sujets infectés par les virus HIV, la PPC survient lorsque le taux de lymphocytes T4 est inférieur à 250 par $\mathrm{mm}^{3}$. Des facteurs non immunitaires, comme la composition du surfactant, ou des infections intercurrentes pourraient aussi avoir un rôle dans l'augmentation de la virulence du parasite. Chez le rat, les corticoïdes induisent une augmentation du taux de phospholipides dans le liquide de lavage bronchoalvéolaire (LBA) [26] ; ce taux diminue au cours de la pneumocystose $[26,27]$. La fréquente concomitance $\mathrm{m} / \mathrm{s} n^{\circ} 6$ vol. 6 , juin 90

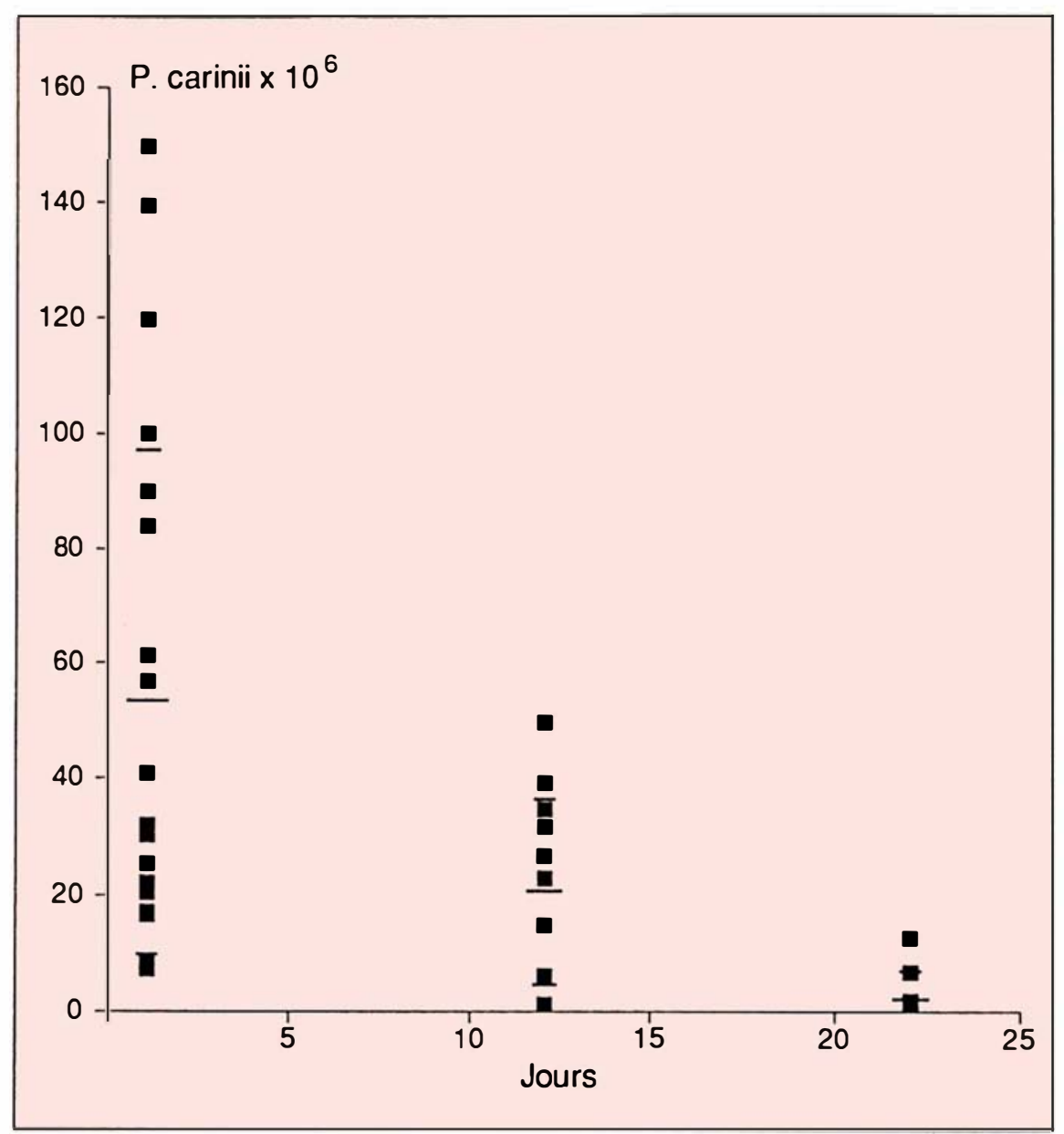

Figure 3. Évolution du parasitisme par Pneumocystis carinii chez le lapereau. Nombre de formes kystiques (carrés noirs) présentes dans le poumon de lapins spontanément parasités et sacrifiés le jour du sevrage (28e jour), 40 et 50 jours après la naissance. Les animaux n'étaient soumis à aucun traitement. Moyennes : traits horizontaux longs; écarts types : traits horizontaux courts.

du parasitisme par $P$. carinii et de l'infection par cytomégalovirus a fait évoquer des interactions entre ces deux micro-organismes [28].

La pneumocystose spontanée chez un hôte " intact ", le lapereau non traité par des corticoïdes [24] permet maintenant d'envisager les rapports hôteparasite entre Pneumocystis et son hôte sous un angle nouveau. Quand et comment les lapereaux.s'infestentils ? La voie de transmission généralement admise est la voie aérienne ; le seul mécanisme bien établi est le passage d'un animal infesté à un autre animal (m/s $n^{\circ} 5$, vol. 1, p. 104). Cependant, la constatation de l'infection par $P$. carinii chez des animaux de quelques jours dont la mère est peu parasitée (résultats non publiés) permet de suggérer que la voie transplacentaire puisse constituer une voie supplémentaire d'infection. Ainsi, le lapereau développe une primo-infection à $P$. carinii qui devient patente au moment du sevrage et dont la résolution spontanée s'accompagne de la séroconversion et de l'acquisition d'une immunité. La survenue ultérieure d'un état immunodépressif permet la réactivation de formes latentes de $P$. carinii et le développement de la PPC [23]. Il est intéressant d'ajouter que ni les souris ni les rats au sevrage ne développent de PPC spontanée.

L'élucidation des facteurs qui conditionnent la survenue de la PPC au 
moment du sevrage chez le lapereau présenteront un grand intérêt. Il est remarquable que durant cette période, pouvant correspondre à un affaiblissement transitoire des défenses de l'organisme, le seul agent opportuniste qui parvienne à proliférer est $P$. carinii. En effet, d'après nos recherches microbiologiques et parasitologiques, les maladies dues à d'autres agents ne sont pas plus fréquentes au sevrage qu'à d'autres périodes de la vie. Des facteurs immunologiques et non immunologiques peuvent être en cause. Des anticorps maternels anti-Pneumocystis transmis aux lapereaux par voie transplacentaire ou galactogène pourraient jouer un rôle protecteur transitoire s'estompant au sevrage. Cependant les mères lapines sont souvent séro-négatives et nous n'avons pas réussi (résultats préliminaires) à mettre en évidence d'anticorps spécifiques dans le sérum des lapereaux avant le sevrage. Malheureusement, les réactifs permettant d'étudier les sous-populations lymphocytaires du lapin ne sont pas encore disponibles. En ce qui concerne les facteurs non immunologiques, il est certain que des variations dans la composition du surfactant pourraient expliquer la prolifération du parasite (étude en cours).

L'infection naturelle $\mathrm{du}$ lapereau semble mimer la primo-infection humaine dont témoigne la séroconversion vis-à-vis de $P$. carinii rapportée chez l'enfant durant ses 2 à 4 premières années de vie $[5$, vol. $I$, p. 35] et qui serait la preuve de l'acquisition d'immunité. La pneumocystose chez l'homme immunodéprimé serait aussi le résultat de la réactivation endogène de $P$. carinii [1]. Il est donc évident que la connaissance des rapports hôte-parasite chez un hôte potentiellement immunocompétent, comme le lapereau, permettrait d'aborder l'étude des facteurs impliqués dans la transition saprophyte-pathogène de Pneumocystis.

\section{Aspects physiopathologiques}

L'habitat de $P$. carinii est l'alvéole pulmonaire. Les trophozoïtes s'attachent spécifiquement et très étroitement aux pneumocytes de type 1

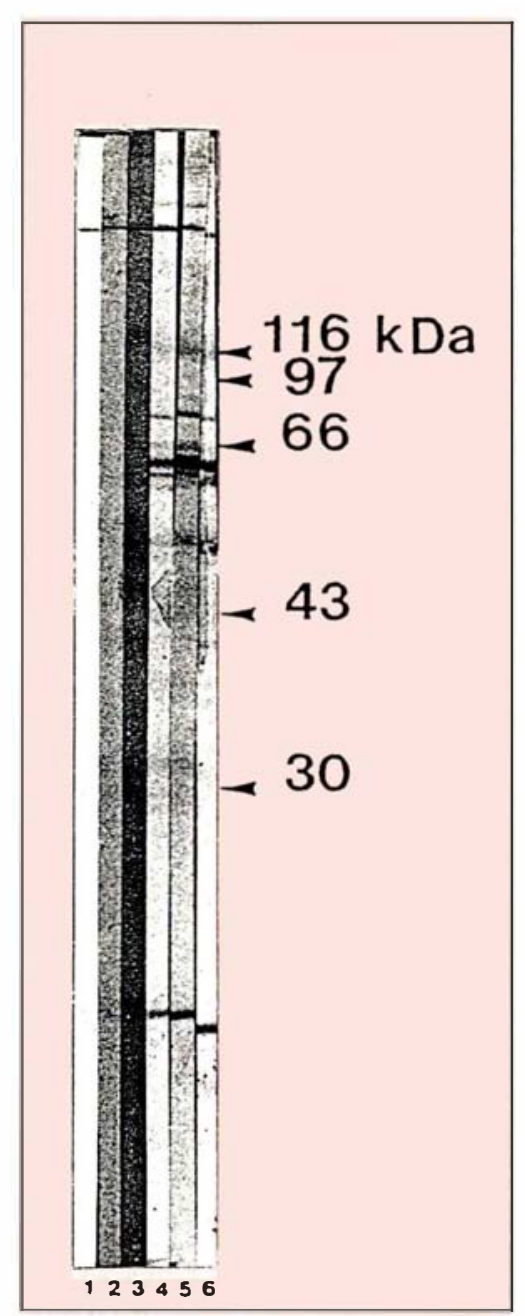

(figure 1) qui ne montrent pas chez le lapin de changements dégénératifs. Chez le rat, de telles lésions cellulaires sont observées lorsque le parasitisme est très intense [1]. Chez le lapin, les systèmes membranaires des pneumocytes de type 2 apparaissent parfois dilatés et leurs cytoplasmes et nucléoplasmes raréfiés. La région alvéolo-capillaire apparaît souvent littéralement couverte par des trophozoïtes (figure 5). L'augmentation de la perméabilité capillaire serait, chez le rat, le premier changement lors de l'infection par $P$. carinii [1].

La PPC induit chez le lapereau au sevrage une augmentation de la concentration de triglycérides sériques et une diminution du rapport cholestérol-HDL/cholestérol total [24]. Ces anomalies sont probablement à rattacher à une inhibition de la lipoprotéine-lipase par le tumor necrosis factor, comme cela a déjà été rapporté au cours d'autres infections, parasitaires ou non [29].

\section{La réponse immune}

Des nombreuses maladies du système immunitaire, congénitales ou acquises, font le lit de la pneumopathie à $P$. carinii chez l'homme, qui apparaît comme la conséquence d'une désorganisation globale des systèmes de défense.

Des anticorps sériques antiPneumocystis sont détectés fréquemment chez les individus normaux. Au cours de la PPC, leur taux le plus souvent diminue. Des anticorps spécifiques de type IgA [30] et IgG [31] sont retrouvés complexés à la surface du parasite. Le transfert passif d'un anticorps monoclonal anti-Pneumocystis chez des furets infectés provoque un ralentissement dans l'évolution de l'infection [25]. Les souris athymiques n'ont pas d'anticorps antiPneumocystis. La réponse humorale semble donc être thymodépendante. Ces animaux sont partiellement protégés par le transfert passif de lymphocytes $\mathrm{T}$ [32].

Cependant, l'immunité humorale n'est pas responsable à elle seule de la protection de l'hôte vis-à-vis de Pneumocystis. L'infiltrat macrophagique dans les poumons infectés, la phagocytose des parasites observée in vivo et in vitro et la supériorité des
Figure 4. Réponse humorale (IgG) du lapin vis-à-vis de l'infection Les chiffres en dessous de carinit. piste correspondent au nombre de semaines à partir du sevrage. Chaque apin prélevé une fois par semaine. mon de rat. Des anticorps sont faible ment détectables dès la troisième semaine. Ils réagissent vis-à-vis de fractions antigéniques de 115, 75, 65 et $45 \mathrm{kDa}$. La réponse humorale est corrélée à la baisse du taux de parasitisme (voir figure 3 ). 


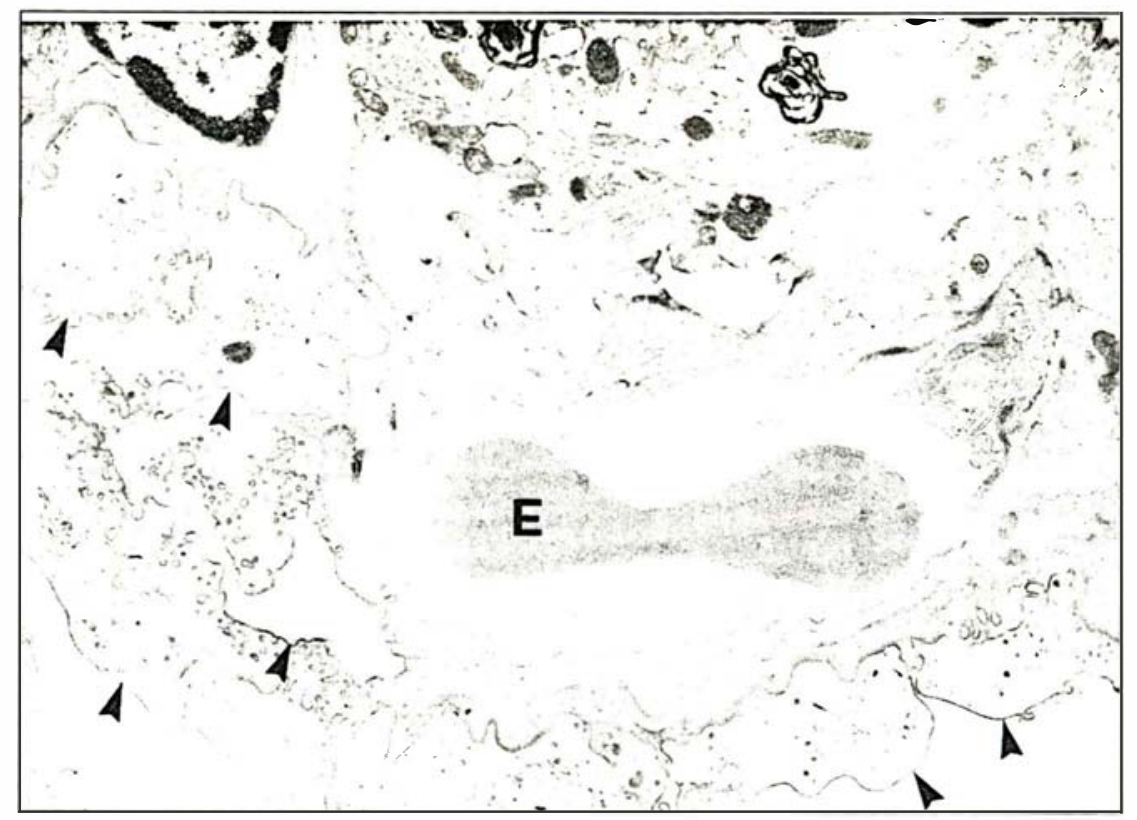

Figure 5. Pneumocystis carinii dans le poumon. Des nombreux trophozoites (pointes de flèche) tapissent la lumière alvéolaire en couvrant la surface d'un capillaire qui apparaît en section transversale contenant un érythrocyte (E). x 12 768. Hôte expérimental : lapin. Les perturbations des échanges gazeux caractéristiques de la pneumocystose semblent ainsi avoir une traduction ultrastructurale.

corticoïdes sur la ciclosporine (un inhibiteur des lymphocytes $\mathrm{T}$ ) [5, vol. $I, p$. 75] pour induire une pneumocystose chez le rat, illustrent l'importance des mécanismes non spécifiques pour la défense de l'hôte. L'atteinte des lymphocytes $\mathrm{CD}^{+}$, par la désorganisation globale de la réponse immunitaire qu'elle entraîne chez les patients infectés par HIV, semble être un facteur essentiel pour la transformation saprophytepathogène de $P$. carinii.

En dépit des difficultés pour obtenir un antigène pur et des obstacles inhérents au statut immunitaire des hôtes expérimentaux, différentes fractions antigéniques cibles de la réponse humorale ont été identifiées et plusieurs équipes ont obtenu des anticorps monoclonaux dirigés contre ces protéines [30]. Ceux-ci autorisent certains auteurs à suggérer qu'existent des différences entre les souches de Pneumocystis trouvées dans le poumon de différents mammifères.

$m / s n^{\circ} 6$ vol. 6 , juin 90
Une des principales difficultés que pose actuellement la recherche sur $P$. carinii est l'obtention de parasites vivants et dépourvus de tissus de l'hôte. Les animaux de laboratoire traités par des corticoïdes ont permis des progrès très importants sur les plans fondamental et pratique. La pneumocystose du lapereau, non traitée par des corticoïdes, permet d'aborder plus aisément les rapports hôte-parasite et la transformation saprophyte-pathogène de $P$. carinii. Des efforts sont nécessaires pour la mise au point de cultures in vitro plus performantes permettant d'obtenir des quantités importantes de parasites purs pour des études immunologiques et de biologie moléculaire, de tester in vitro l'activité de molécules anti- $P$. carinii et, peut-être, de mieux comprendre la biologie et le cycle de cet important opportuniste

\section{Summary}

Pneumocystis carinii : a challenge for biologists

Pneumocystis carinii is the agent of a severe interstitial pneumonitis in immunosuppressed patients, especially with AIDS. The taxonomic position of $P$. carinii remains unclear. Trophozoites closely resemble protozoa, whereas the cyst cell wall presents staining and structural similarities with fungi. Corticosteroid-treated or athymic rats and mice were until now the only source of $P$. carinii and they made possible the evaluation of drugs for the treatment of $P$. carinii pneumonitis (PCP). Corticosteroid-untreated rabbits at weaning develop a spontaneously resolving PCP. Important perturbations of cell mediated immunity and probably, unspecific defence mechanisms, play a role in the pathogeny of PCP. The knowledge of host-parasite relationships in a corticosteroid-untreated host, such as the rabbit at weaning, makes it possible to approach the saprophyte-pathogen transformation of $P$. carinii. In vitro, $P$. carinii development was reported in cultures with and without feeder cells and in explant cultures of rabbit lung. The last system allows to maintain the parasite during several weeks.

\section{TIRÉS A PART}

E. Dei-Cas. 